\title{
Quantitative 3D characterization of solidification structure and defect evolution in Al alloys
}

\author{
C. Puncreobutr ${ }^{1}$, P.D. Lee ${ }^{2}$, R.W. Hamilton ${ }^{1}$, and A.B. Phillion ${ }^{3}$ \\ ${ }^{1}$ Department of Materials, Imperial College London, Prince Consort Road, London, SW7 2BP, UK \\ ${ }^{2}$ School of Materials, The University of Manchester, Oxford Road, Manchester, M1 7HS, UK \\ ${ }^{3}$ School of Engineering, The University of British Columbia, Kelowna, BC, V1V 1V7, Canada
}

\begin{abstract}
Geometrically complex, as-cast, aluminum alloy components offer substantial economic and design advantages as compared to many thermo-mechanical processing routes. However, the occurrence of potential defects, including porosity, intermetallics and hot-tears, remains an issue. Using these three defects as examples, recent developments in the methodologies for quantifying defect evolution and microstructure using 3D images captured in situ via X-ray microtomography are discussed. It is demonstrated that 3D quantification techniques can provide significant new insight into the mechanisms controlling defect formation, and how microstructure morphology affects component performance.
\end{abstract}

\section{$\underline{\text { Introduction }}$}

Aluminum alloy usage is continually increasing in the automotive and aerospace sectors, driven by the demand for improved fuel economy and performance through weight reduction [1]. Studies show a $10 \%$ reduction in vehicle weight reduces greenhouse gas emissions by 6-8\%. Since the automotive and aerospace sectors account for about $22 \%$ of the world's total $\mathrm{CO}_{2}$ emissions [2], any weight reduction will give immediate environmental benefits. Unfortunately, high strength to weight alloys can present processing challenges that increase cost, limiting market penetration. The occurrence of solidification defects - porosity [3, 4], intermetallics [5-7] and hot-tearing [8-10] - is one of the major sources of processing issues.

The presence of porosity and intermetallics can limit final mechanical properties. For example, fatigue life is strongly influenced by pore size, decreasing by a factor of 8 when pores increase from 100 to $600 \mu \mathrm{m}$ in radius $[11,12]$. The in-service failure of cast components, such as automotive wheels and engine blocks, is often dominated by the level of porosity if its size is over $50 \mu \mathrm{m}$, when pores often act as fatigue initiation sites. Intermetallics can have a similar influence [6]. Hot-tearing, where thermal stresses lead to semi-solid cracking during processing is also detrimental since it results in part rejection and alloy substitution [9]. Hot-tears have been shown to nucleate on pores, intermetallics, and oxides [8, 13]. Thus, quantifying these defects formation and growth is of great scientific and industrial interest.

Developments in computational resources combined with advances in X-ray generation and detector technology has led to tremendous improvement over the past two decades in our 
ability to quantitatively characterize metallic-alloy solidification defects. Although X-rays have been used for observing solidification in situ since the 1960's, when Forsten and Miekk-oja [14] studied the progression of a solid-liquid front, it was not until the 1990's that in situ observation of defect formation became more common (e.g. [15, 16]). Since then, advances have been very rapid using both laboratory sources, and especially with the development of third-generation synchrotron X-ray sources, which allow the capture of 3D images with both high spatial $(<1 \mu \mathrm{m})$ [17] and temporal $(<1 \mathrm{~s})$ resolution [18]. Equally importantly has been the development of the algorithms and computational resources for performing the 3D image analysis of the results. All of these factors have combined to provide a unique ability to directly observe pore, intermetallic, and hot-tear formation, allowing new insights into old problems. Quantification of structure and defect morphology has also led to new models of microstructure development $[19,20]$, and will reduce industrial processing costs.

The benefits of 3D imaging are clearly shown when comparing the 2D section (typical of an optical micrograph) in Fig.1(a) with the 3D tomograph of an as-cast A319 sample in Fig.1(b). If only the 2D were analyzed, then one would conclude that the sample contained about 10 pores, with a maximum length of $\sim 300 \mu \mathrm{m}$; the 3D quantification clearly shown that most of these 'separate' pores are actually a single large $(\sim 1100 \mu \mathrm{m})$, tortuous pore. When one compares $2 \mathrm{D}$ and 3D measurement techniques of pore equivalent radius, maximum length and percentage porosity, Fig.1(c-e) [21], only percentage porosity is equivalent. Tynelius et al. [22] and Yi et al. [11] both demonstrated maximum pore length to be the most important factor for fatigue life, 2D measurements can underestimate the value by over a factor of 3 (e.g. Fig.1(d)). However, even in 3D the measurement are not always accurate, as shown by the example in Fig.1 where a new methodology (g) illustrated that the accepted method (f) gives a 34\% overestimate (as detailed later). Hence, new methods of quantification are always being developed. In this paper some of these new methodologies are first detailed, followed by two examples of their application to in situ experiments.

\section{Quantification of 3D Solidification Defects}

Quantifying the observations obtained from 3D in situ observations of solidification is challenging. Firstly, the amount of data generated is large - one experiment can generate a terabyte of data. Secondly, although the human eye has evolved to differentiate patterns, developing code with similar abilities is not straight-forward. Thirdly, the time required for analysis can be considerable. Despite these challenges, a range of both commercial (e.g. Avizo, Matlab, VG Studio Max, Simpleware-ScanIP) and research/open-source (ITK, ImageJ, and inhouse, e.g. $[23,24])$ automated tools have been developed to efficiently process 3D X-ray tomographic images, quantifying the structures within. These tools can provide a range of numeric parameters to characterize 3D images: 


\section{(a) Scalar Measuring Tools}

Basic 3D quantification of solidification defects includes volume fraction $\left(f_{v}=\frac{V_{d}}{V_{T}}\right)$, where $V_{d}$ is the total volume of the defect and $V_{T}$ is the total volume of the 3D image, number density $\left(N_{v}=\frac{n_{d}}{V_{T}}\right)$, where $n_{d}$ is the total number of defects, and defect size distribution, where $N_{v}$ is divided into sub-classes based on the equivalent spherical radius.

\section{(b) Shape Measuring Tools}

The power of 3D imaging is in quantifying the shape of defects. Shape quantification can provide new knowledge regarding both defect formation, and its effect on mechanical performance.

\section{Curvature \& Tortuosity}

Prediction of as-cast porosity in aluminum alloys requires knowledge of the gas pressure within the pore. The pressure difference between the gas and the liquid phase is given by the Young-Laplace equation $\left(\Delta P=2 \gamma_{l g} \bar{\kappa}\right)$, where $\gamma_{l g}$ is the liquid-gas surface energy and $\bar{\kappa}$ is the local mean curvature $\bar{\kappa}=\frac{1}{2}\left(\frac{1}{R_{1}}+\frac{1}{R_{2}}\right)$, where $R_{1}$ and $R_{2}$ are the two principal radii of curvature. As shown by Felberbaum et al. [25], analysis of 3D X-ray tomographic images of Al-4.5wt.\%Cu indicates that a wide range of porosity radii exist in $\mathrm{Al}-\mathrm{Cu}$ alloys, resulting in pores of complex morphology that can be fairly well approximated to cylinders.

Tortuosity refers to the path-length of a flow channel as compared to the straight-line distance between its ends, i.e. $\tau=\tau_{i j}(d \sigma / d \eta)^{2}, \tau_{i j}=\left(\partial \xi_{i} / \partial \sigma\right)\left(\partial \xi_{j} / \partial \sigma\right)$, where $\sigma$ is the length measured along the streamline, $\eta$ is the length measured along axis of channel, and $\xi_{i}$ and $\xi_{j}$ are the local coordinates [26]. During alloy solidification, this quantity will affect a semi-solid material's ability to feed liquid to regions of low pressure and hence is directly related to porosity and hot-tearing. Although tortuosity is in general quite difficult to determine, the 3D image provides a direct means to calculate a tortuosity factor $\tau=\sqrt{\pi\left(X_{\min }+X_{\max }\right) / 2 S}$, where $S$ is the surface area of the pore, and $X_{\min }$ and $X_{\max }$ are the average values of minimum and maximum chessboard distance in $2 \mathrm{D}$ sections respectively [27].

\section{Surface Area Quantification}

Spherical defects pose less of a risk for fatigue initiation as compared to rod or plate-like defects, and thus knowledge of a defect's sphericity can be used to evaluate the effects of processing or chemistry changes on in-service performance. Using 3D images, the surface-tovolume ratio can either be calculated directly, i.e. $S_{A}: V=\frac{S_{A}}{V_{T}}$ where $S_{A}$ is the surface area, or through use of a dimensionless quantity such as the sphericity factor, $\varepsilon$, which is defined as the ratio of the equivalent spherical surface area to the measured surface area, i.e. $\varepsilon=\frac{\sqrt[3]{\left(36 \pi V_{T}\right)^{2}}}{S_{A}}$, and 
provides a measure of a defect's deviation from spherical. Although a classical surface area calculation that counts voxel faces at the boundary between object and background gives an efficient estimation of the surface area, the result is always overestimated [28]. An improved estimation of the surface area can be obtained by an isosurface reconstruction of polygonal surfaces which is triangulated via a Marching Cube algorithm [29].

\section{Spatial Orientation}

Most commercially-available codes determine the $x-y-z$ spatial configuration of a defect through use of an axis-aligned bounding box, i.e. the smallest box with sides parallel to the Cartesian coordinate system that is needed to fully contain the defect. However, as shown in Fig.1(f) and Fig.1(g), this measure overestimates the maximum length, given as the diagonal length. A better approximation is to align the box to the axes of the defect. Principal Component Analysis (PCA) is one method of achieving this - it effectively calculate the object's major axes from the eigenvectors of a covariance matrix based on the $x-y-z$ coordinates of all the voxels making up that object. The technique also enables a defect's orientation to be compared to processing parameters, such as thermal gradient, rolling direction, and/or thermally-induced strains. For example, several of the intermetallics in Fig.1(b) were measured with the standard bounding box diagonal, Fig.1(f), and then via the major diagonal obtained by the PCA method, Fig.1(g). The error using a bounding box aligned to the observers frame of reference is $34 \%$, giving a maximum length of 100 instead of $74.6 \mu \mathrm{m}$. PCA analysis has been successfully applied to all three types of defects $[11,30-32]$.

\section{Quantification Examples}

Application of the methodologies described above are illustrated on two examples, the first demonstrating the quantification of pores and intermetallics, the second, hot-tear formation. The experimental methods are described first followed by results and discussion.

\section{Experimental Methods}

In order to capture the in situ formation of porosity and intermetallics in three dimensions, an apparatus is required that will rotate a sample with high precision and speed (synchronized with the camera and allowing radiographs to be acquired at small angular increments over $360^{\circ}$ for tomographic reconstruction), while contained in a furnace with controlled heating/cooling. For hot-tearing, control and measurement of displacement and load is also required. To enable these experiments, a bespoke rig, the so-called P2R semi-solid tester shown in Fig.2, was developed. This apparatus was designed and constructed with the following technical specifications: load application of $\pm 1-500 \mathrm{~N}$, specimen extension of $\pm 0-150 \mathrm{~mm}$, and stepped or continuous specimen rotation over $\pm 0-360^{\circ}$ at speeds up to $1 \mathrm{~Hz}$ while placed within a furnace capable of reaching $1000^{\circ} \mathrm{C}$. The encoded accuracy was $0.1 \mathrm{~N}, 200 \mathrm{~nm}$, and $0.001^{\circ}$.

Using the apparatus outlined above, solidification defects were investigated in two alloy systems. For the porosity study, an A319 alloy with an enhanced Fe level (Al-7.5Si-3.5Cu- 
$1.2 \mathrm{Fe}$, wt.\%) was used, together with a cooling rate of $0.36{ }^{\circ} \mathrm{C} / \mathrm{s}$ from the liquid state. For the hot-tearing study, an alloy of $\mathrm{Al}-15 \mathrm{wt} . \% \mathrm{Cu}$ was selected, with and without an addition of $9.6 \mathrm{vol} \% \mathrm{Al}_{2} \mathrm{O}_{3}$ particulate sized in the range $10-30 \mu \mathrm{m}$. The hot-tearing experiments were performed by heating a pre-solidified sample up to $560^{\circ} \mathrm{C}$ and then pulling in tension at a constant cross-head displacement of $10 \mu \mathrm{m} / \mathrm{s}$. The experiments were all performed on Beamline I12 at the Diamond Light Source using a $53 \mathrm{keV}$ monochromatic X-ray source. Radiographic images were acquired at a spatial resolution of $12.22 \mu \mathrm{m}$ using a Phantom camera with 800x600 pixels and $5.5 \mathrm{~ms}$ integration time. This camera configuration one 3D tomographic image every $4 \mathrm{~s}$ and had the ability to acquire a maximum of $243 \mathrm{D}$ images during each experiment.

\section{$\underline{\text { Results }}$}

\section{(a) Pores and Intermetallic Morphology}

The mechanisms by which pores nucleate $[7,19,33]$ and interact with each other and the developing primary phase remains an area of debate. Traditional metallography of the final structures can only indicate if potential nuclei are co-located, rather than proving the chronological porosity nucleation and growth sequence. One on-going area of interest is the influence of intermetallics on pores, specifically if pores nucleate on intermetallics during the solidification of Fe containing Al alloys [4, 33-35]. For example, looking qualitatively at the colocation of the intermetallics and pores in Fig.1(b), it would appear that there is strong support for this hypothesis. However, by analyzing the time resolved in situ 3D observations, the exact physical relationship, as well as the growth sequence of each phase, can be observed and analyzed.

The results of such an analysis are shown in Fig.3, where the rate of growth and sphericity values for both pores and intermetallics are plotted as a function of temperature in an A319 alloy. By extrapolating the rate of growth back to volume fraction of zero, the nucleation events can be estimated. For this set of processing conditions, the larger pores nucleate before (higher T) the intermetallics. Further, there is a significant decrease in the sphericity of the porosity at the same point as when it impact onto the intermetallics. From these observations we can hypothesize that the pores grow more quickly on encountering intermetallic, spreading along their surface, perhaps because the interfacial energy between the gas and solid is lower than between the gas and liquid aluminium. The intermetallics therefore acted to alter the morphology of the larger pores, but in this case did not nucleate them. However, closer examination of Fig.3 shows that there are a number of smaller pores that appear to nucleate after the intermetallics. Unfortunately the resolution of these ultra-fast tomography results was insufficient to resolve if they nucleated on the intermetallics.

\section{(b) Evolution of Damage during Hot-Tearing}

In the field of hot-tearing, one area that is not well understood is the influence of particulate reinforcement on damage initiation and growth during metal matrix composite (MMC) solidification. Although the addition of particulates increases strength and reduces micro- 
segregation and the size of micro-porosity (among other benefits), hot-tearing susceptibility is significantly increased in MMC [36] as compared to "standard" aluminum alloys. In Fig.4, 3D renderings of the hot-tear formation in (a) semi-solid $\mathrm{Al}-15 \mathrm{Cu}$ and (b) semi-solid $\mathrm{Al}-15 \mathrm{Cu}-$ 9.6vol.\% MMC at a fraction solid of 0.75 are shown just prior to final fracture. As can be seen, the crack geometry (colored as red) appears to be significantly more distributed in the $\mathrm{Al}-15 \mathrm{Cu}$ specimen, almost reaching the ends of the gauge region, whereas the MMC specimen contains a crack localized to a small volume of material. This difference in the two materials is indicative of their deformation behaviour.

In Fig.4(c), the volume fraction of both internal damage and surface-connected damage is quantified as a function of strain, for the two specimens. As can be seen, there are significant differences in hot-tearing behaviour. In the $\mathrm{Al}-15 \mathrm{Cu}$ specimen, the initial as-cast porosity is relatively high and well distributed throughout the gage length (Inset I). The application of tensile load results in substantial growth of internal damage, along void nucleation and subsequent coalescence. At $\boldsymbol{\varepsilon} \sim 0.35$ a peak is observed since the internal deformation-induced void structure has reached the surface of the specimen. After this point, further deformation appears as surface-connected damage until failure occurs at $\varepsilon \sim 0.68$ (Inset II). In contrast, hottearing in the MMC specimen occurs only near the center of the gage length. As can be seen, the initial specimen appears to contain very little as-cast porosity (Inset III) ${ }^{1}$. Furthermore, relatively little internal damage accumulates during the hot-tearing process but instead the deformation is accommodated via surface-connected crack formation until failure occurs at $\varepsilon \sim 0.35$ (Inset IV).

Based on the 3D in situ observations, the differences in hot-tearing behaviour are thought to be due to the relative ease of liquid flow with and without particulate matter. In the $\mathrm{Al}-15 \mathrm{Cu}$ specimen, the tensile load appeared to enable liquid to flow towards the center of the gage to accommodate strain with void nucleation occurring only when liquid feeding was eliminated. Once the voids were connected to the surface, the pressure on the interdendritic liquid was released, and the liquid was sucked back into the bulk to shrink the porosity. In contrast, the structure of the MMC specimen had much more difficulty to feed the strained region since the presence of the particulate appeared to both block the interdendritic flow path and increase the bulk viscosity of the liquid. Instead of the strain being distributed over a wide mushy zone, the stress increases with little ductility in the semi-solid, leading to hot-tearing at very low strains (but high stress), with a fracture surface more like a brittle failure.

The evolution of crack morphology during hot-tearing is also important for understanding the mechanisms of semisolid deformation. Although this work is currently ongoing using PCA for the specimens presented in Fig.4, the required effort is considerable and is not yet complete. In Fig.5, the evolution in crack morphology during the semi-solid deformation of an AA5182 alloy is presented. This figure, based on work from a previous study [32], provides the change in

${ }^{1}$ This observation may simply be due to the resolution limit of the tomography scan since the particulates are known to block pore growth as well as to act as heterogeneous nucleation sites and create very fine microporosity. 
void orientation in relation to the loading direction at three different levels of strain $(\varepsilon=0,0.09$, 0.39) from a specimen in which the deformation was "interrupted" to acquire the 3D tomographic images. Insets I and II show equal-area pole figures of individual void orientations at $\varepsilon=0$ and $\varepsilon=0.39$ in which points at the center (perimeter) correspond to voids having a major axis parallel (perpendicular) to the loading direction. The results indicate that the initial as-cast material contains voids with random orientation (i.e. between 0-90 deg.). With increasing strain, the morphology evolves considerably. Although, the voids seem to firstly grow in all directions, they then become preferentially oriented perpendicular to the loading direction (i.e. 90 deg. and lying near the circumference of the pole plot) at $\varepsilon=0.39$, with very few voids having a major axis oriented less than $45 \mathrm{deg}$. to the loading direction. As discussed in Phillion et al. [32], although the presence of the liquid phase influences crack growth by adding randomness to the crack propagation path, deformation ultimately orients perpendicular to the loading direction to utilize the maximum strain energy for new surface creation. Furthermore, the liquid enables crack propagation along predefined paths, leading to low ductility at high fraction solid.

\section{Conclusions}

The combination of in situ 3D observation of $\mathrm{Al}$ alloy solidification structure using synchrotron X-ray micro-tomography and advanced defect-analysis quantification techniques provides new insights into the mechanisms controlling porosity, intermetallic, and hot-tear formation. With this new insight, new processing route can be designed that will optimize material behavior and final mechanical properties, improving our ability to efficiently create near-net-shape cast components for transportation and aerospace applications.

\section{Acknowledgements}

The authors would like to acknowledge the provision of beamtime and help from the scientists at the Diamond Light Source's I12 Beamline, especially T. Connolley, R.C. Atwood and M. Drakopoulos, as well as P. Rockett for help with the design of the P2R Rig. The authors also would like to thanks EPSRC (EP/F001452/1), M-ORS and the Thai Government Scholarship for financial support. 


\section{Figures}

Fig. 1. Comparison of 2D and 3D solidification defects: (a) Segmented 2D optical micrograph of porosity and intermetallics in an A319 aluminium alloy; (b) True 3D morphology of the same sample; (c) Equivalent radius, $R_{\text {avg, }}$ (d) Maximum length, $L_{\max }$, and (e) Percentage porosity, $P \%$ comparison between metallographic (2D) and tomographic (3D) analysis for the same alloy (after Lee et al. [21]); (f) $x-y-z$ bounding box measurement of the intermetallic from Fig.1(b); and $(\mathrm{g})$ Intermetallic shape and measure after rotation to principal plane via PCA.

(a)

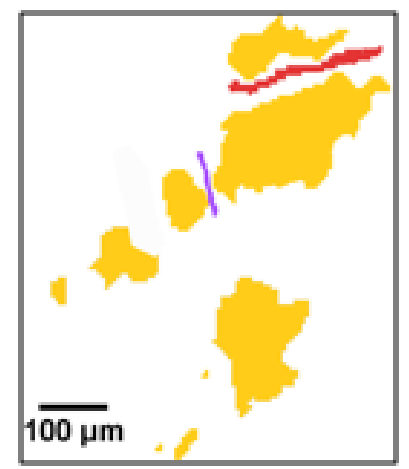

(b)

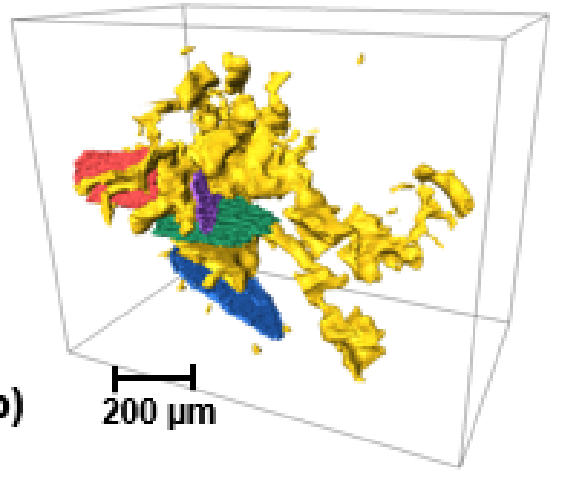

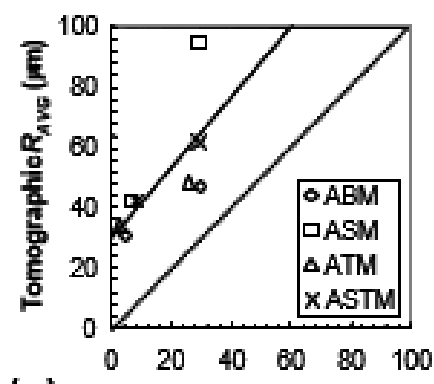

(c)

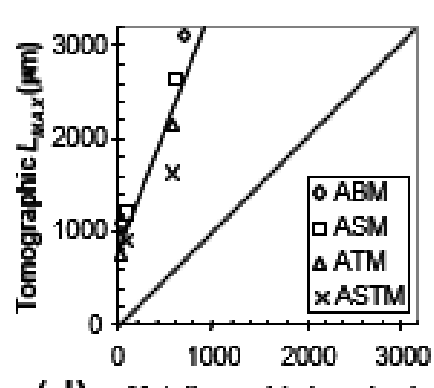

(d) Metallographic $L_{\max }(\mu \mathrm{m})$

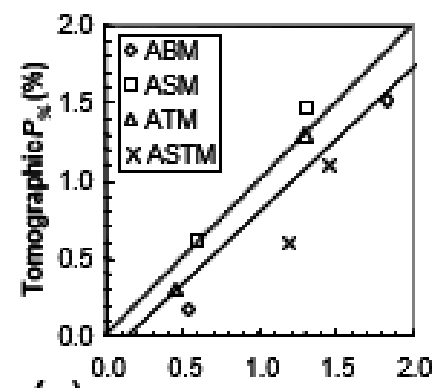

(e) (f)

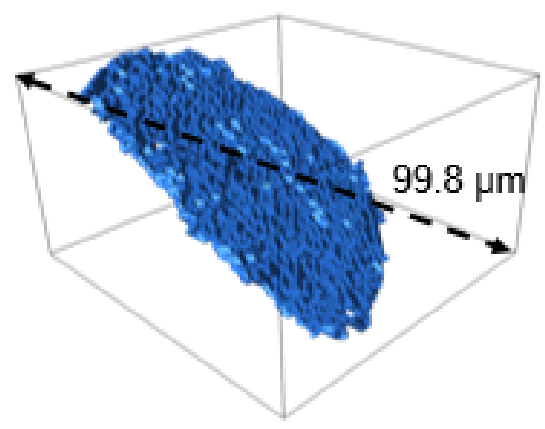

Mean of 4 intermetallics $=71 \pm 23 \mu \mathrm{m}$ (g)

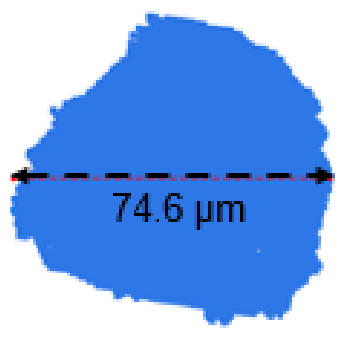

Mean of 4 intermetallics $=56 \pm 18 \mu \mathrm{m}$ 
Fig. 2. P2R experimental rig for real-time tomographic observations of solidification mechanisms and semi-solid deformation.

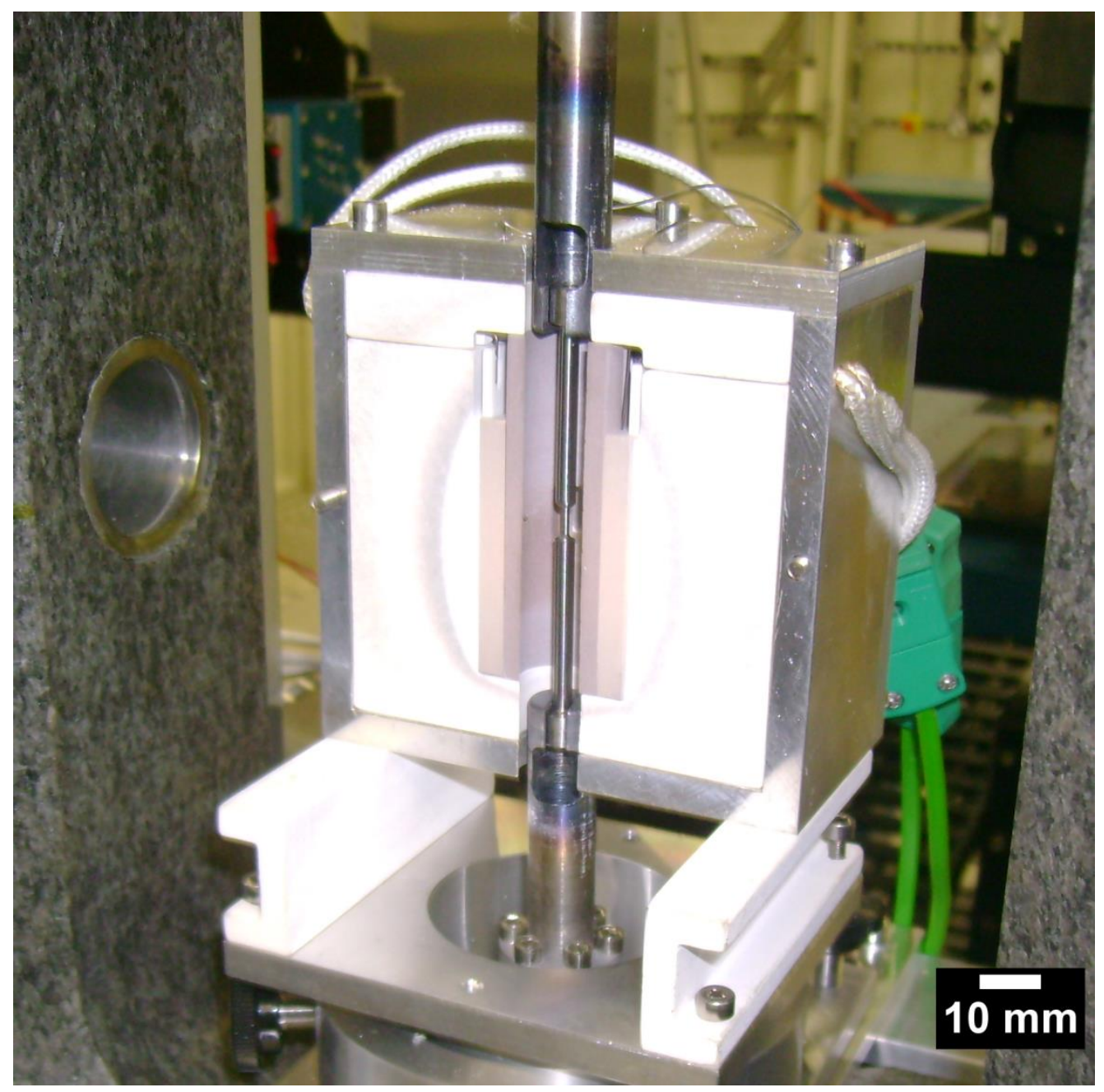


Fig. 3. Real-time tomographically quantified pore evolution (volume and sphericity) as a function of temperature with Insets I-V showing the typical pore morphology at four different time steps. For comparison purposes, the intermetallic evolution from in situ radiography experiments is provided.

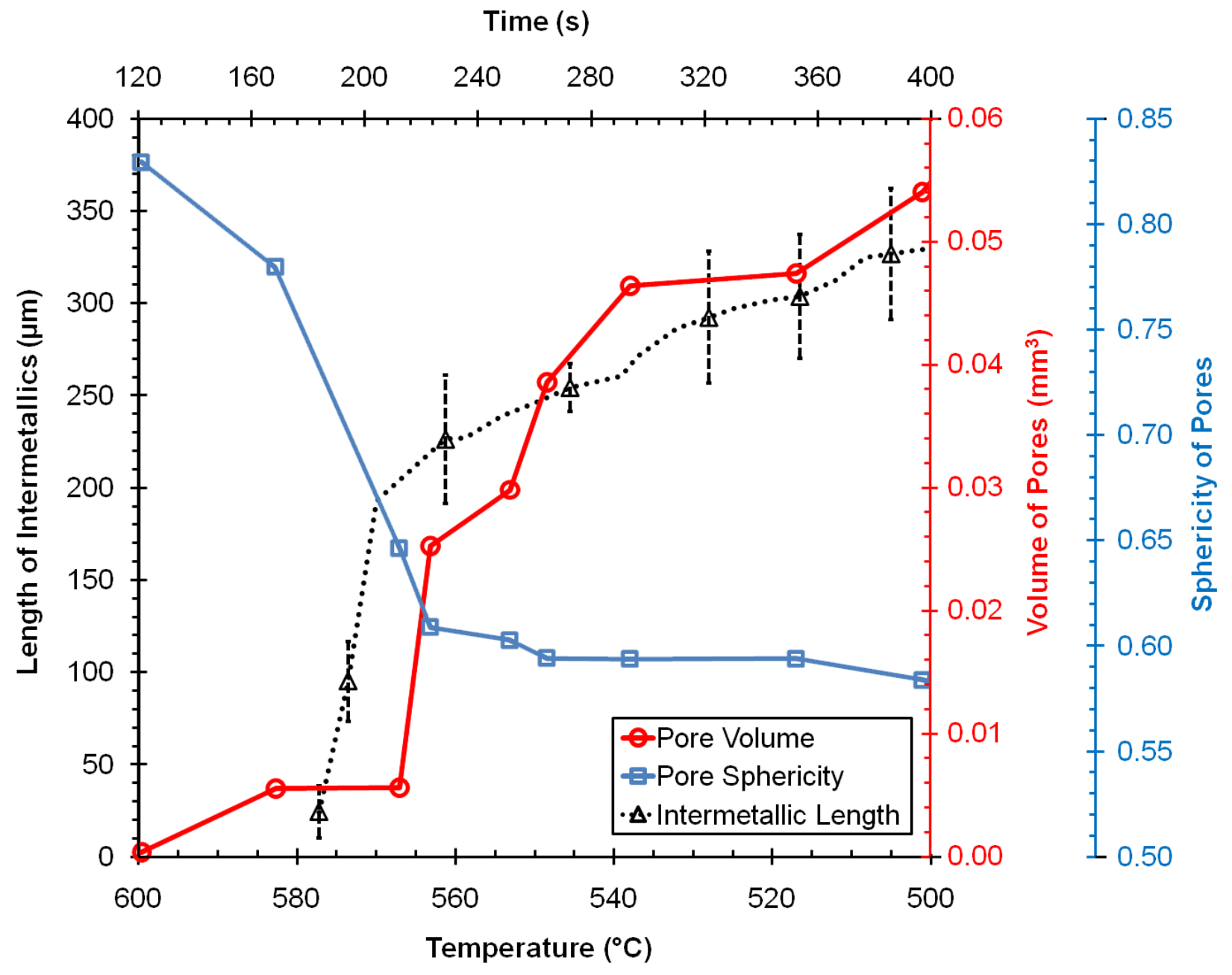


Fig. 4. 3D rendering of the solidification structure near final hot-tearing in (a) $\mathrm{Al}-15 \mathrm{Cu}$ and (b) $\mathrm{Al}-15 \mathrm{Cu}-9.6 \mathrm{vol} . \% \mathrm{MMC}$ as well as the quantified evolution in (c) the internal damage volume fraction and surface connected cracking. Inset I, II (III,IV) show longitudinal micrographs of Al$15 \mathrm{Cu}(\mathrm{Al}-15 \mathrm{Cu}-9.6 \mathrm{vol} . \% \mathrm{MMC})$ at $\varepsilon=0$ and $0.65(0$ and 0.35$)$.

(a)

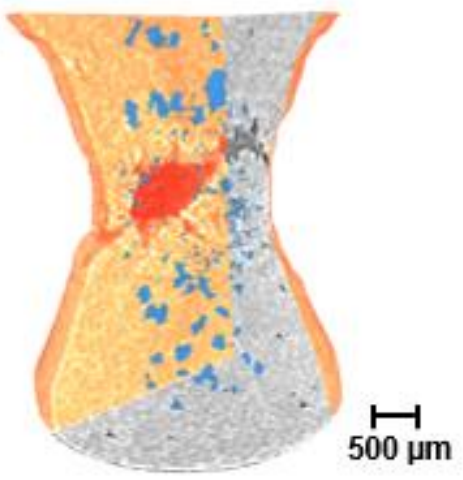

(b)

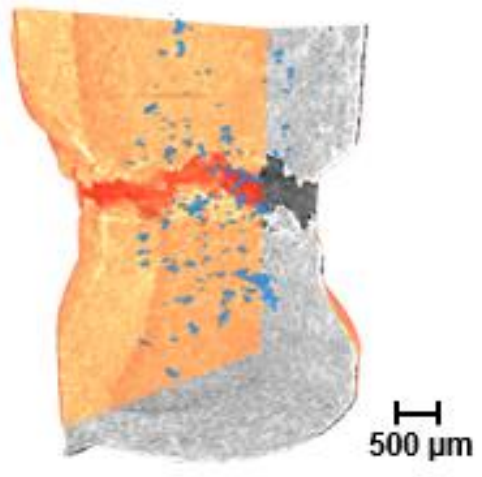

(c)

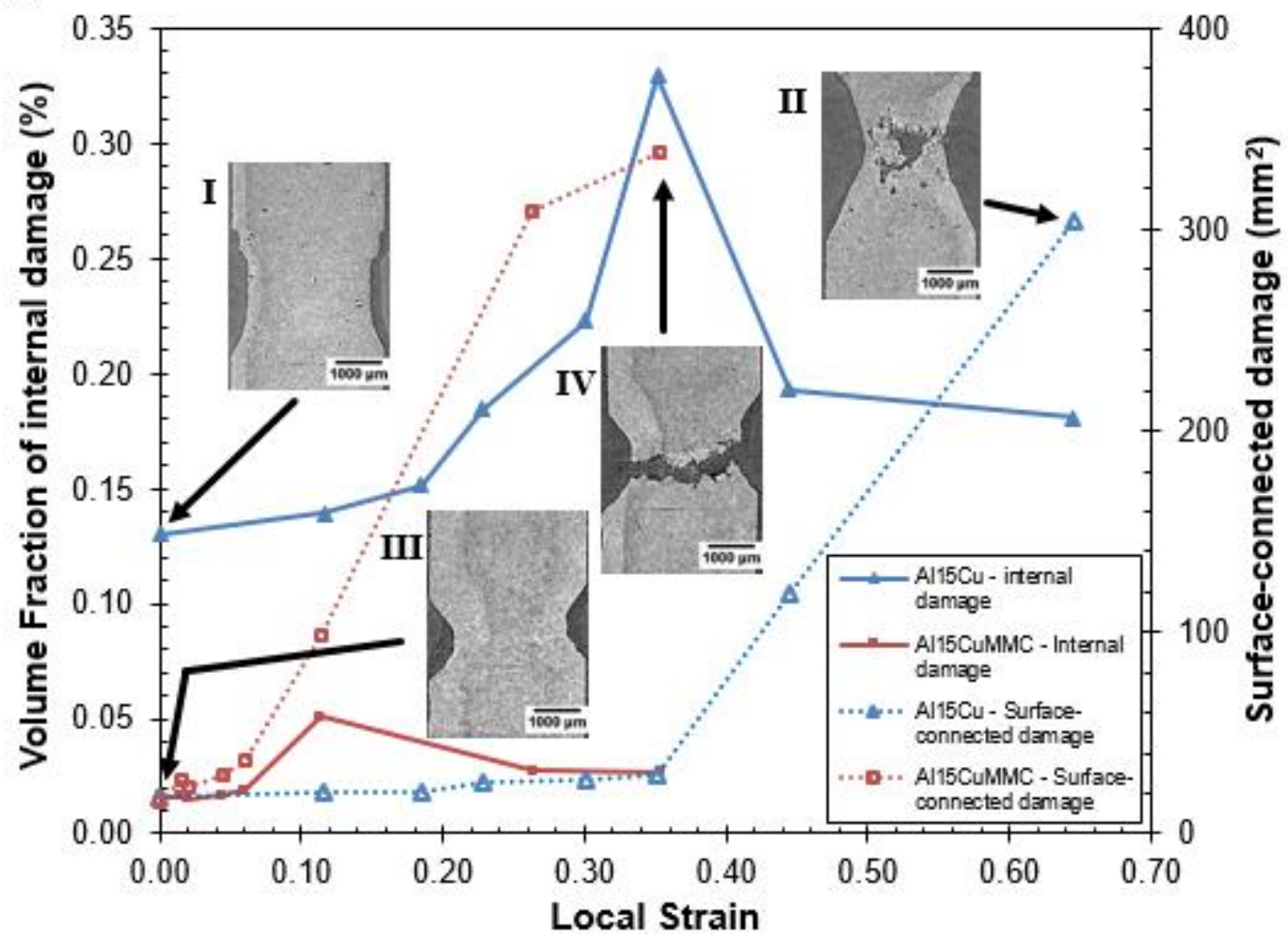


Fig. 5. Variation in internal damage orientation/morphological texture with increasing strain. Insets I and II show pole figures with the individual orientation of each void (>1000voxels) plotted for $\varepsilon=0$ and 0.39 , while Inset III shows a 3D rendering of the damage $(9 \mu \mathrm{m}$ voxel resolution).

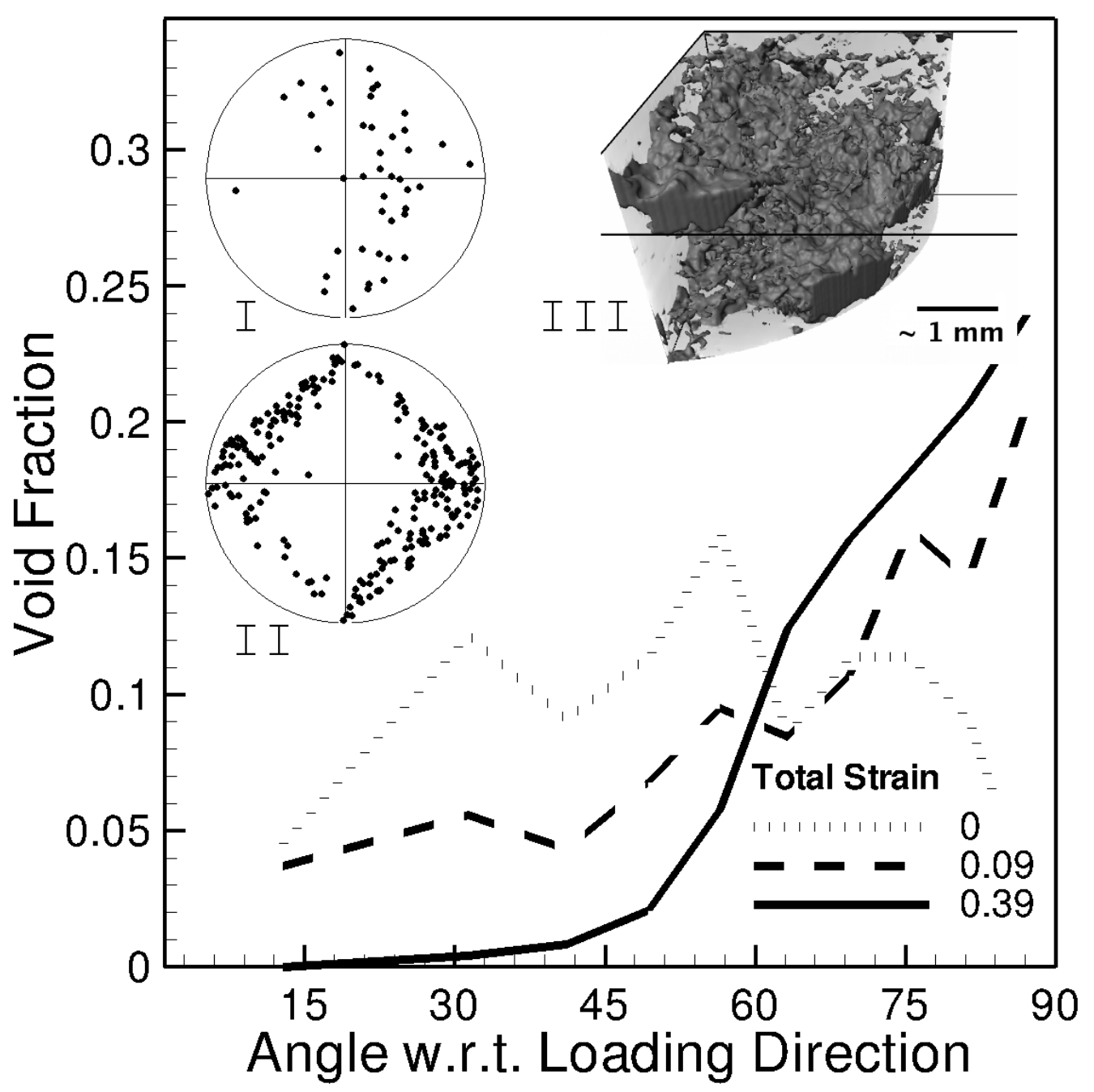




\section{References}

1. J. Hirsch, Materials Transactions JIM, 52 (5) (2011), pp. 818-824.

2. The International Energy Agency (IEA), "CO2 emissions from fuel combustion: highlights," 2010, http://www.iea.org/co2highlights/.

3. M.J. Couper, A.E. Neeson and J.R. Griffiths, Fatigue Fract Eng M, 13 (3) (1990), pp. 213-227.

4. P.D. Lee and J.D. Hunt, Acta Metall Mater, 45 (10) (1997), pp. 4155-4169.

5. L. Lu and A. Dahle, Metall Mater Trans A, 36 (3) (2005), pp. 819-835.

6. J.Z. Yi, et al., Mat Sci Eng A, 386 (1-2) (2004), pp. 396-407.

7. J. Wang, et al., Scripta Mater, 60 (7) (2009), pp. 516-519.

8. Y.F. Guven and J.D. Hunt, Cast Metals, 1 (2) (1988), pp. 104-111.

9. $\quad$ A.B. Phillion, et al., Acta Metall Mater, 59 (4) (2011), pp. 1436-1444.

10. $\quad$ S. Terzi, et al., Scripta Mater, 61 (5) (2009), pp. 449-452.

11. J. Yi, et al., Metall Mater Trans B, 37 (2) (2006), pp. 301-311.

12. J.F. Major, AFS Trans, 105 (1990), pp. 901-906.

13. I. Farup, J.M. Drezet and M. Rappaz, Acta Metall Mater, 49 (7) (2001), pp. 1261-1269.

14. J. Forsten and H.M. Miekk-oja., J. Inst. Met., 95 (1967), pp. 143-145.

15. D. Stegemann, W. Reimche and J. Schmidbauer, The European Journal of Nondestructive Testing, 1 (3) (1992), pp. 107-117.

16. P.D. Lee and J.D. Hunt, "A Model of the Interaction of Porosity and the Developing Microstructure," in Modeling of Casting, Welding, and Advanced Solidification Processes London, UK, 10-15 September 1995, pp. 585-592.

17. F. Beckmann, et al., Adv Eng Mater, 9 (11) (2007), pp. 939-950.

18. R. Mokso, F. Marone and M. Stampanoni, AIP Conference Proceedings, 1234 (1) (2010), pp. 87-90.

19. P.D. Lee and J.D. Hunt, Acta Metall Mater, 49 (8) (2001), pp. 1383-1398.

20. J. Wang, et al., J Appl Phys, 107 (6) (2010), pp. 061804.

21. P.D. Lee, et al., "The Effect of Metallurgical Variables and Porosity on the High Cycle Fatigue Behaviour ofAluminium Alloy A319," in COM, Vancouver, B.C., 24-27 August 2003, pp. 457-472.

22. K.Tynelius, J.F.Major and D.Apelian, AFS Trans, 101 (1993), pp. 401-413.

23. R.C. Atwood, et al., Scripta Mater, 51 (11) (2004), pp. 1029-1033.

24. S. Yue, et al., Acta Biomater, 7 (6) (2011), pp. 2637-2643

25. M. Felberbaum, "Porosity in Aluminum Alloys : Visualization, Characterization, and Modeling," (PhD thesis, EPFL, 2010). 
26. F.A.L. Dullien, Porous media: fluid transport and pore structure, 2nd ed. (London: Academic Press, 1992).

27. C.D. Cantrell, Modern mathematical methods for physicists and engineers, 1st ed. (Cambridge: Cambridge University Press, 2000).

28. I. Nystroem, et al., "Area of and volume enclosed by digital and triangulated surfaces," in Medical Imaging 2002: Visualization, Image-Guided Procedures, and Display, San Diego, CA, 17 May 2002, pp. 669-680.

29. W.E. Lorensen and H.E. Cline, "Marching cubes: A high resolution 3D surface construction algorithm," in Computer graphics and interactive techniques, New York, NY, 28 July - 2 August 1987, pp. 163-169.

30. D.L. McDowell, et al., Eng Fract Mech, 70 (1) (2003), pp. 49-80.

31. J. Allison, et al., JOM- J Min Met Mat S, 58 (11) (2006), pp. 28-35.

32. A.B. Phillion, et al., Metall Mater Trans A, 39 (10) (2008), pp. 2459-2469.

33. J. Campbell, ISI Publication, 110 (1968), pp. 18-26.

34. N. Roy, A. Samuel and F. Samuel, Metall Mater Trans A, 27 (2) (1996), pp. 415-429.

35. C.M. Dinnis, J.A. Taylor and A.K. Dahle, Mat Sci Eng A, 425 (1-2) (2006), pp. 286-296.

36. A. Mortensen and I. Jin, Int Mater Rev, 37 (1992), pp. 101-128. 